\title{
Diseño y evaluación de un tejido sintético para la simulación de nudos y suturas mediante laparoscopia
}

\author{
Design and evaluation of a synthetic tissue for simulation of laparoscopic \\ knotting and suturing
}

\author{
Liliana Cuevas-López ${ }^{1}$ Claudia Marcela Echeverri-Gómez²
}

1 Médica, cirujana general, Hospital Universitario San Ignacio, Facultad de Medicina, Pontificia Universidad Javeriana, Bogotá,
D.C., Colombia
2 Médica, residente de Cirugía General, Pontificia Universidad Javeriana, Bogotá, D.C., Colombia

Tercer puesto en el Simposio Nacional del Residente Quirúrgico, XLIV Congreso Nacional "Avances en Cirugía", Cartagena, Colombia, agosto de 2018

\section{Resumen}

Introducción. La adquisición de habilidades psicomotoras dentro del proceso de formación de un residente de Cirugía requiere de una exposición repetida y previa a la práctica en el paciente. Las competencias para suturar y anudar en laparoscopia son parte de éstas y, usualmente, la primera exposición a dichos procesos se logra en centros de simulación, donde la práctica mejora el desempeño final en los residentes.

Encontrar en el mercado un producto que permita simular dicha práctica de forma fácil y asequible es difícil en nuestro medio, principalmente por altos costos; por esta razón, desarrollamos un tejido semisintético, fabricado en casa, económico, reutilizable, replicable, de consistencia similar al intestino y fácil de utilizar en el ambiente de simulación laparoscópica.

Materiales y métodos. Se realizó un estudio observacional descriptivo con 25 cirujanos laparoscopistas que evaluaron el tejido en mención mediante una práctica de sutura y anudado por laparoscopia, por medio de una encuesta semiestructurada de percepción tipo Likert.

Resultados. La calificación global de la experiencia tuvo una mediana de 9 en una escala visual análoga de o a Io; en cuanto a las características específicas del tejido, la percepción fue que el tejido permitía realizar con mucha facilidad una sutura simple para el $68 \%(n=17)$, sutura continua para el $52 \%(n=13)$ y anudado intracorpóreo para el $76 \%$ (n=I9) de los cirujanos.

Las características más frecuentemente calificadas como mejores por los cirujanos fueron consistencia y firmeza, así como una progresión en la facilidad para realizar las tareas propuestas. Las características que limitaron o dificultaron el desarrollo de la práctica, nombradas por los cirujanos, fueron resistencia ocasional, distinta consistencia en algunas áreas del tejido, partículas, y cuatro de ellos no encontraron ninguna característica desfavorable. Conclusión. El tejido propuesto cumple las características buscadas al realizarlo y brinda un modelo de simulación útil para mejorar el aprendizaje de nudos y suturas por laparoscopia.

Palabras clave: laparoscopía; obtención de tejidos y órganos; simulación; enseñanza mediante simulación de alta fidelidad; educación médica; programas de postgrado.

Fecha de recibido: 8/06/2018 - Fecha aceptación: 28/08/2018

Correspondencia: Liliana Cuevas-López, MD, Carrera 7 N 40-62, Bogotá, D.C., Colombia, Teléfono: (571) 594-6161.

Correo electrónico: Icl92@hotmail.com

Citar: Cuevas-López L, Echeverri-Gómez CM. Diseño y evaluación de un tejido sintético para la simulación de nudos y suturas mediante laparoscopia. Rev Colomb Cir. 2018;33:362-70. https://doi.org/10.30944/20117582.83

Este es un artículo de acceso abierto bajo una Licencia Creative Commons - BY-NC-ND https://creativecommons.org/licenses/by-nc-nd/4.0/deed.es 


\begin{abstract}
Introduction: The training of a surgical resident should include practical exposure to knowledge that has been translated into motor skills. The skills of suturing and knotting by laparoscopy are usually the first exposure in simulation centers, where the possibility of repeated practice improves the final performance in the residents. Finding in the market a product that simulates real tissue, like the intestine, easily and affordably, is difficult in our environment. We developed a synthetic tissue that was manufactured at home, which is economic, reusable, with consistency similar to the intestine, and easy to use in the laparoscopic simulation environment.

Materials and methods: A descriptive observational study was carried out with 25 laparoscopic surgeons who evaluated the mentioned tissue through laparoscopic suturing and knotting, by means of a semi-structured perception survey.

Results: The overall score of the experience had a median of 9 on a visual analog scale from o to Io in regard to the specific characteristics of the tissue, the perception that it allows a simple and continuous suture to be performed easily, was $68 \%(n=17)$ and $52 \%(n=13)$ respectively, and to perform laparoscopic intracorporeal knotting with much ease was $76 \%(n=19)$.
\end{abstract}

Conclusion: the proposed tissue fulfills the desired characteristics and provides a useful simulation model to improve the learning of knotting and suturing by laparoscopy

Keywords: laparoscopy; tissue and organ procurement; simulation; high fidelity simulation training; education, medical; health postgraduate programs

\section{Introducción}

La realización de nudos y suturas por laparoscopia es una habilidad compleja. Para aprender y desarrollar adecuadamente esta destreza, se requiere de información teórica, seguida de una práctica en la que se repita dicho aprendizaje I-3. En el caso de la sutura y el anudado laparoscópicos, hay una variedad de perfiles tisulares, la mayoría costosos, con implicaciones éticas y logísticas para su desarrollo y utilización (como los modelos animales); algunos tienen fragilidades inherentes al tejido e, incluso, por sus características pueden llegar a proporcionar una percepción alejada del tejido real, con riesgo de desarrollar una integración cognitiva y psicomotora defectuosa ${ }^{4,5}$.

Independientemente de la clase de tejido que se use durante la simulación, este debe estar orientado a generar un escenario de gran fidelidad, mediante el cual se logre adquirir habilidades psicomotoras específicas y mayor experiencia en la sala de operaciones. Debe ser una herramienta práctica diseñada para el fortalecimiento educativo y la repetición adecuada, que genere un impacto en la autoconfianza del residente, y que se dé en un marco económico, ético, factible y que permita su reutilización ${ }^{6-12}$. En el momento, no existen en la literatura estudios comparativos para determinar cuál es el mejor material para el aprendizaje, ni se han especificado con precisión sus características ideales. La información disponible se basa en modelos descriptivos de los distintos materiales, con beneficios individuales y experiencias adquiridas según cada uno.

Existe la necesidad de obtener un tejido sintético de bajo costo, reutilizable, ajustado a los diferentes niveles y tipos de residencia quirúrgica, que simule los tejidos reales, que abra las puertas para un currículo de simulación de alto nivel y que, a su vez, sea extrapolable a diversos programas. 
El objetivo de este estudio fue implementar un modelo de tejido de gran fidelidad, económico, reutilizable y fácil de utilizar, que permita simular las habilidades de sutura y anudado manual laparoscópico, en un ambiente de evaluación objetiva y validado por cirujanos laparoscopistas experimentados.

\section{Materiales y métodos}

Inicialmente, durante un año se hicieron diversas pruebas de acuerdo con el soporte de la sutura y el anudado, la búsqueda del grosor, el aspecto y el color como características principales, hasta que se encontró el tejido ideal para este proyecto.

La fabricación de este perfil tisular incluye una preparación a base de glicerina, coloide, agua, tinte de color piel y una mezcla de los mismos, en cantidades proporcionales, que se llevan al horno microondas por 20 segundos según la cantidad preparada. Luego, la mezcla caliente se vierte sobre la cara anterior y posterior de la felpa, en un recipiente de loza de vidrio, el cual se lleva a refrigeración y se espera a que la consistencia -inicialmente líquida de la mezcla-se fije y se solidifique. Al final de la preparación, la mezcla coloide se ha integrado a la felpa.

Una vez el material se encuentra firme y sólido, se despega de la loza y se le da una forma cilíndrica que simule el intestino. Su fabricación tarda 30 minutos, aproximadamente, son suficientes 6 horas de refrigeración y no necesita de temperaturas extremas o procesos industriales. El costo aproximado de un tejido de $I 0$ x $10 \mathrm{~cm}$ es de COP\$ 2.00o.

La validación inicial se hizo por medio de una práctica piloto con ocho residentes y tres docentes de cirugía, con aceptación del tejido antes de ser sometido a la evaluación descrita en este estudio. Una vez aprobado, se inició un estudio observacional descriptivo y recurrente, en el cual participaron 25 cirujanos laparoscopistas de Bogotá, quienes asistieron al centro de simulación de la Pontificia Universidad Javeriana para hacer una prueba estandarizada en el tejido.
Durante la asistencia, se proporcionó un tejido de $10 \mathrm{~cm}$ a cada cirujano, el cual estaba previamente moldeado en forma cilíndrica para simular el intestino. Este molde se acomodó en el simulador de laparoscopia (pelvitrainer) y se le practicó un corte lineal para simular la herida por suturar (figura I). A cada cirujano se le entregó una sutura trenzada 3.o, previamente cortada entre 15 y $20 \mathrm{~cm}$ (según preferencia del cirujano) para hacer una sutura simple, el anudado y una sutura continua.

La práctica se hizo en un simulador de laparoscopia, con cámaras de I.08o pixeles, utilizando como instrumentos laparoscópicos un portaagujas y una pinza Maryland (figura 2). Antes de iniciar la práctica, se dio a conocer el propósito del estudio a todos los participantes, las características por evaluar y el proceso de evaluación del tejido. Se dieron instrucciones sobre el uso del tejido, las suturas y el anudado. Al finalizar la práctica, se envió por correo electrónico un cuestionario semiestructurado a cada uno de los participantes para que, de forma anónima, evaluaran cuatro aspectos principales: estética, sensación general, utilidad y la actitud en general hacia el tejido (apéndice I).

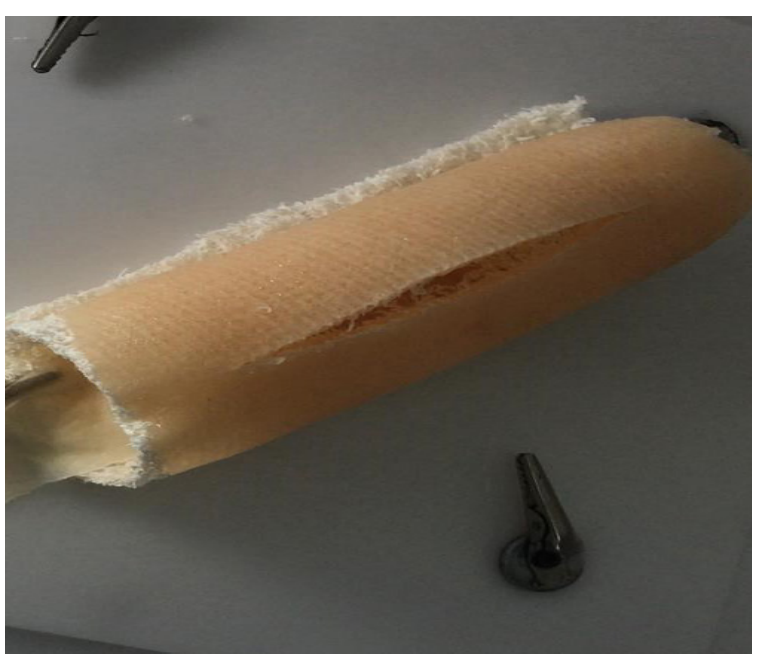

Figura 1. Tejido con herida lineal, moldeado cilíndricamente y ajustado al simulador de laparoscopia (pelvitrainer) 
Las variables demográficas analizadas fueron edad, sexo, lateralidad y años de experiencia del cirujano laparoscopista.

\section{Análisis estadístico}

Las variables cuantitativas se analizaron mediante medidas de tendencia central y dispersión; y las variables cualitativas, por medio de frecuencias relativas (porcentajes) y proporciones. Se utilizó el programa Excel $^{\circledR}$ (2016, Microsoft) para la organización de variables y el cálculo de las proporciones y porcentajes, y el programa Atlas Ti, para el análisis cualitativo de los datos.

\section{Aspectos éticos}

Este estudio es de tipo descriptivo y cualitativo; por lo tanto, no se hace ninguna intervención sobre los participantes. Se clasifica como un estudio "sin riesgo", según la Resolución oo8430 de

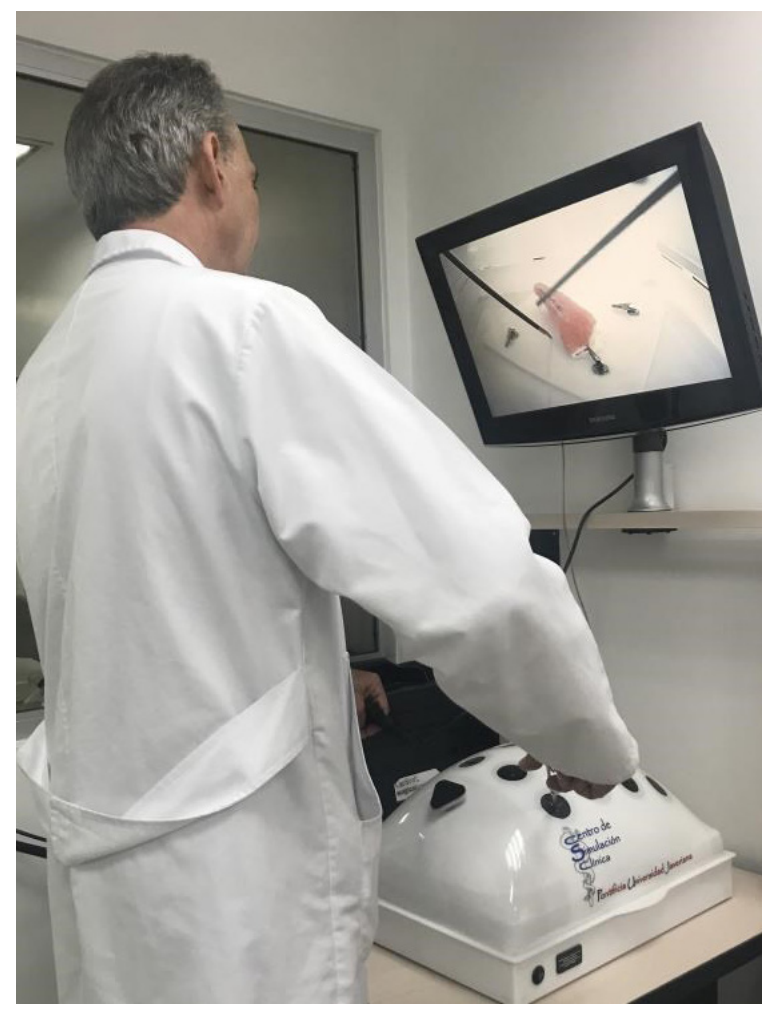

Figura 2. Práctica en el simulador de laparoscopia (pelvitrainer)
I993 del Ministerio de Salud de Colombia, prevaleciendo en esta investigación el criterio del respeto a la autonomía, la dignidad y la protección de los derechos, la información y el bienestar de los sujetos incluidos en el estudio. Además, en este estudio se respetaron las normas éticas que tienen su principio en la última versión oficial de la declaración de Helsinki.

\section{Resultados}

Participaron 25 cirujanos laparoscopistas, la mayoría fueron hombres diestros. Los datos demográficos de los participantes se muestran en la tabla I. En cuanto al tipo de sutura, II (44\%) practicaron ambas suturas y I4 (56\%), únicamente sutura continua.

Se utilizó una escala visual análoga de o a Io, para evaluar los apartados de estética, sensación general y la experiencia global como parte del área de actitud.

Con respecto a la estética, se evaluaron el color, la consistencia y la textura, obteniendo calificaciones promedio de 9,8 y 8 , respectivamente. En relación con la impresión general y la experiencia global del uso del tejido, la calificación promedio fue de 8 . Los rangos y las medianas se muestran en la tabla 2.

Tabla 1. Datos demográficos de los cirujanos encuestados

\begin{tabular}{llcc}
\hline \multirow{2}{*}{ Características } & \multicolumn{2}{c}{ Encuestados $(\mathbf{n = 2 5 )}$} & $\%$ \\
\hline Edad (años) & Rango & 29 a 56 & \\
& $\begin{array}{l}\text { Promedio } \\
\text { Desviación } \\
\text { estándar }\end{array}$ & 39 & \\
& Femenino & 3 & 12 \\
& Masculino & 22 & 88 \\
\hline Lateralidad & Diestro & 24 & 96 \\
& Zurdo & 0 & 0 \\
& Ambidextro & 1 & 4 \\
\hline Años de & Rango & 2 a 28 & \\
experiencia & Promedio & 10 & \\
& Desviación & 8 & \\
& estándar & & \\
\hline
\end{tabular}


En relación con la utilidad, I4 (56\%) de los cirujanos afirmaron que el tejido usado en la práctica simulaba una sensación de tejido intestinal real; específicamente en relación con el tipo de sutura, I7 (68\%) respondieron que el tejido permitía hacer una sutura simple y, I3 (52\%), una sutura continua, con mucha facilidad. De los 25 cirujanos, i9 (76\%) opinaron que el anudado intracorpóreo laparoscópico se podía hacer con mucha facilidad (figura 3).

Al explorar la actitud frente al uso del tejido, $20(80 \%)$ de los cirujanos estuvieron muy a favor de tener más prácticas en este tipo de tejido, 5 (20\%), algo a favor, y ninguno, poco a favor.

En cuanto a lo que más les agradó y les permitió hacer una mejor simulación de sutura y anudado laparoscópico, la mayoría de los cirujanos encuestados nombraron más de una característica en sus respuestas. Las más frecuentes fueron la consistencia y la firmeza, así como una progresión en la facilidad para hacer las tareas propuestas; otras descritas fueron textura, movi- lidad de los bordes, color, fácil paso de la aguja y la sutura. Cinco de ellos confirmaron una sensación muy parecida a la del tejido real y algunos refirieron que el modelo se comportaba de forma muy adecuada o, inclusive, mejor que otros tejidos usados previamente. Sobre las características que limitaron o dificultaron el desarrollo de la práctica de simulación de sutura y anudado laparoscópico, la mayoría afirmó que las dificultades no se asociaron con el tejido, sino con factores relacionados con el simulador de laparoscopia o el instrumental empleados. Otras características desfavorables nombradas, fueron resistencia ocasional, distinta consistencia en algunas áreas del tejido (heterogeneidad) y partículas; cuatro de los cirujanos no encontraron ninguna característica desfavorable (tabla 3).

\section{Discusión}

Los resultados de este estudio demuestran que el tejido fabricado fue evaluado de forma positiva por los cirujanos laparoscopistas encuestados.

Tabla 2. Evaluación de la estética, sensación general y experiencia global del uso del tejido en la práctica de simulación

\begin{tabular}{lccccc}
\hline & Color & Consistencia & Textura & $\begin{array}{c}\text { Impresión } \\
\text { general }\end{array}$ & $\begin{array}{c}\text { Experiencia } \\
\text { global de } \\
\text { anudado y sutura }\end{array}$ \\
\hline Rango & 7 a 10 & 4 a 10 & 4 a 10 & 5 a 10 & 5 a 10 \\
Promedio & 9 & 8 & 8 & 8 & 8 \\
Mediana & 8 & 8 & 8 & 8 & 9 \\
\hline
\end{tabular}

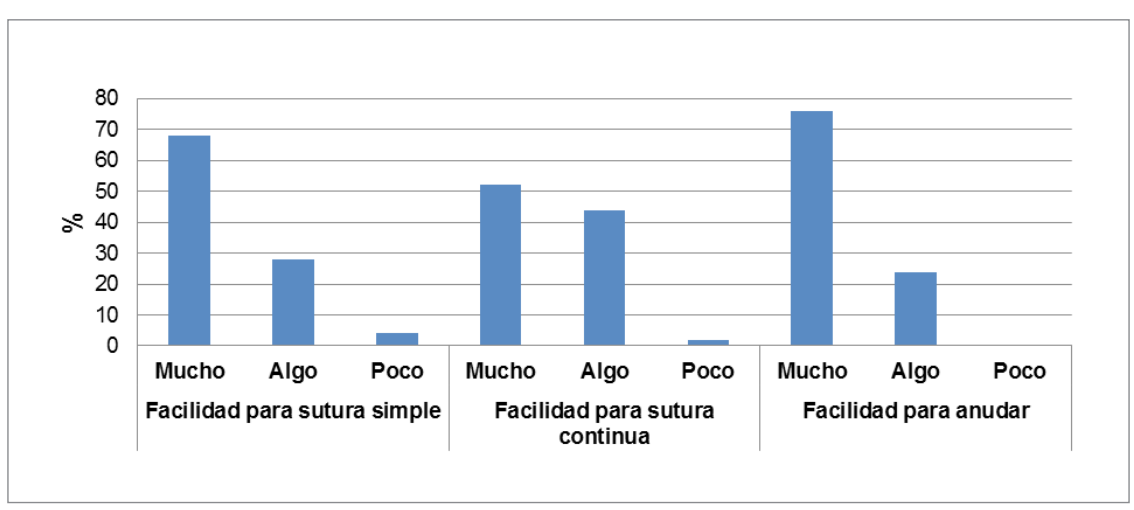

Figura 3. Utilidad del tejido para sutura y anudado 
Tabla 3. Características agradables y desagradables percibidas por los cirujanos

\begin{tabular}{|c|c|c|c|}
\hline \multicolumn{4}{|l|}{ Características } \\
\hline \multicolumn{2}{|l|}{ Más agradables } & \multicolumn{2}{|c|}{ Menos agradables } \\
\hline Consistencia & $\mathrm{n}=8$ & $\begin{array}{l}\text { Factores } \\
\text { asociados con } \\
\text { el simulador de } \\
\text { laparoscopia o } \\
\text { el instrumental }\end{array}$ & $n=7$ \\
\hline $\begin{array}{l}\text { Aumento de la facilidad } \\
\text { para de sutura y anudado } \\
\text { de forma progresiva con } \\
\text { la práctica }\end{array}$ & $n=6$ & Resistencia & $n=6$ \\
\hline Firmeza & $n=7$ & & \\
\hline $\begin{array}{l}\text { Mejor que otros tejidos } \\
\text { o modelos usados } \\
\text { previamente }\end{array}$ & $n=5$ & $\begin{array}{l}\text { Distinta } \\
\text { consistencia }\end{array}$ & $n=5$ \\
\hline $\begin{array}{l}\text { Sensación similar al } \\
\text { intestino }\end{array}$ & $\mathrm{n}=5$ & & \\
\hline Movilidad de los bordes & $\mathrm{n}=4$ & Ninguna & $n=4$ \\
\hline Color & $n=4$ & & \\
\hline Textura & $n=4$ & Partículas o & $n=3$ \\
\hline $\begin{array}{l}\text { Fácil paso de la sutura } \\
\text { y anudado }\end{array}$ & $n=3$ & tragmentos & \\
\hline
\end{tabular}

El modelo desarrollado fue capaz de simular un tejido real en los ejercicios de sutura y anudado intracorpóreo laparoscópico; es un tejido asequible, repetible, reusable y de bajo costo.

Desde el inicio de los programas de medicina y especializaciones, el método de Halsted (I889) ha sido el patrón de enseñanza en las diferentes escuelas ${ }^{6}$. Sin embargo, con los recortes económicos, las implicaciones éticas, la disminución en el tiempo quirúrgico y las exigencias de parte de los pacientes, cada vez se acorta más el tiempo de exposición de los residentes a la interacción con pacientes.

Esto ha incrementado la dificultad del aprendizaje de habilidades quirúrgicas y modificado este modelo inicial, lo que genera la necesidad de implementar espacios de simulación quirúrgica para complementar la práctica clínica y el aprendizaje de dichas habilidades ${ }^{1,13-16}$. Los programas de residencia están encargados de inte- grar la simulación en su currículo quirúrgico. Lo ideal es iniciar con una práctica en modelos simulados las veces suficientes para dominarlas por completo y, además, perfeccionarlas en la sala de cirugía ${ }^{17}$.

Dentro del proceso de la adquisición de habilidades psicomotoras, Fitts y Posner describieron tres fases del aprendizaje para que cualquier persona pueda adquirir una habilidad. La primera es la fase cognitiva, en la cual se intelectualiza el conocimiento técnico y se debe entender cómo se realiza el procedimiento; la segunda fase es la integrativa, en la cual se traslada el conocimiento a una habilidad motora, y esta fase se alcanza mediante práctica y retroalimentación (el estudiante aún necesita pensar cómo se realiza el procedimiento para lograrlo con éxito); la tercera fase es la autónoma, en la cual el desempeño es óptimo y el estudiante no siente la necesidad de pensar en cómo realizar el procedimiento ${ }^{6}$.

Teniendo en cuenta estas fases, la habilidad de suturar y anudar en cirugía laparoscópica, idealmente, debe alcanzarse en la primera y la segunda fase en un escenario simulado y escalonado, de forma tal que, al momento de ser desarrolladas en el paciente durante una cirugía, se esté en la tercera fase y se haya logrado escalar por medio de una curva de aprendizaje ${ }^{15}$.

Según lo referido por la experiencia de los cirujanos evaluadores, nuestro tejido permite simular la sutura y el anudado laparoscópicos como transición al ámbito quirúrgico que, más que reemplazar al modelo animal, lo complementa. Esto encaja muy bien dentro de este modelo de aprendizaje y se puede implementar en cualquier especialidad donde las habilidades de sutura y anudado sean un requisito.

En relación con el material ideal para la adquisición de una habilidad técnica, como es suturar y anudar por laparoscopia, lo correcto es que sea resistente al desgarro (que puede producirse al paso de una aguja de sutura y al corte de los márgenes de la incisión al hacer el nudo) y que, a su vez, pueda simular la elasticidad y la textura intestinales ${ }^{6,18}$. 
En nuestro modelo, según las características descritas como más favorables para la práctica, el tejido propuesto en este estudio ofrece las condiciones ideales referidas en la literatura para este tipo de simulación; incluso, al analizar algunas características desfavorables anotadas, como las partículas o los fragmentos, no las consideramos desfavorables, dado que en la realidad, muchas veces el cirujano se ve enfrentado a suturar segmentos del tubo digestivo impregnados de restos alimentarios y con soluciones de continuidad que dificultan su visibilidad, tal como lo describieron nuestros participantes.

Entre los métodos utilizados previamente para el aprendizaje de estas habilidades, se han utilizado tejidos animales y sintéticos. En cuanto a los tejidos animales, la calidad se reduce progresivamente con el uso continuo debido a su naturaleza perecedera, pueden tener implicaciones éticas y la logística de su uso puede ser compleja, dada la necesidad de medidas higiénicas por su manipulación. Los tejidos sintéticos son, en su mayoría, muy costosos y algunos pueden estar lejos de simular los tejidos reales. La fabricación del tejido propuesto en esta investigación fue sencilla y permitió varios usos. La portabilidad y el montaje no requirieron mayor logística y no tuvo implicaciones éticas o peligros para el practicante.

Actualmente, el mercado cuenta con modelos o tejidos cuyos precios oscilan entre COP $\$ 40.000$ y COP\$ I20.00o, lo cual es un costo elevado si se pretende cumplir con las necesidades de todos los residentes y de una forma repetitiva. El costo de nuestro tejido es radicalmente menor que el de los modelos de sutura disponibles comercialmente $y$, al permitir varios usos, incrementa su rentabilidad.

Las limitaciones de nuestro estudio radican en que la muestra de cirujanos que evaluaron el tejido fue pequeña, dado que logísticamente el acceso a los horarios de un gran número de cirujanos puede ser difícil. A pesar de ser un estudio descriptivo, se logró el objetivo principal y consideramos que implementar un modelo como este requiere de una primera fase en la que se apruebe su uso. En un futuro, se deben hacer diversas pruebas con este tejido, de forma que se pueda comparar directamente con el modelo intestinal.

\section{Conclusión}

Su fácil uso, las características propias del material, el costo y la naturaleza rentable de los materiales, junto con una fácil elaboración, incluso por el estudiante, hacen que este tejido sea adecuado cuando se desea una alternativa asequible y transicional durante todo el proceso de la adquisición de la habilidad de sutura y anudado laparoscópicos.

\section{Agradecimientos}

A Isabel del Socorro Moreno-Luna, psicóloga con maestría en Epidemiología Clínica de la Pontificia Universidad Javeriana, por sus consejos y asesoría.

Al Centro de Simulación Clínica de la Pontificia Universidad Javeriana, por permitir la práctica en sus instalaciones.

\section{Referencias}

I. Meyers MO, Meyer AA, Stewart RD, Dreesen EB, Barrick J, Lange PA, Farrel TM. Teaching technical skills to medical students during a surgery clerkship results of a small group curriculum. J Surg Res. 20II;166:I7I-5. doi: I0.IOI6/j.jss.20I0.05.0I9

2. Weeks D, Kasdan ML, Wilhelmi BJ. An inexpensive suture practice board. Eplasty. 2015;499-502.

3. Acton RD, Chipman JG, Gilkeson J, Schmitz CC. Synthesis versus imitation: Evaluation of a medical student simulation curriculum via objective structured assessment of technical skill. J Surg. 20I0;67:I73-8. doi: IO.IOI6/j.jsurg.20I0.02.0II

4. Andreatta PB, Marzano DA, Curran DS, Reynolds RK. Evaluation of a precise and measurable model for learning laparoscopic tissue handling. Urol Surg. 20I4;9:283-7. doi: I0.I097/SIH.0000000000000036

5. Zhang L, Grosdemouge C, Arikatla VS, Ahn W, Sankaranarayanan $\mathrm{G}$, Jones $\mathrm{D}$, et al. The added value of virtual reality technology and force feedback for surgical training imulators. Work. 20I2;4I:2288-92. doi: I0.3233/ WOR-20I2-0453-2288

6. Mason KF. Changes in the wind. Compos Technol. 2004;IO:26-3I. doi:IO.IO2I/jfo529I30 
7. van Empel PJ, Verdam MGE, Huirne JA, Bonjer HJ, Meijerink WJ, Scheele F. Open knot-tying skills: Resident skills assessed. J Obstetrics Ginecol Res. 2013;39:IO30-6. doi: IO.IIII/jog.I2II

8. Nagy KK, Roberts RR, Smith RF, Joseph KT, An GC, Bokhari F, et al. Transmediastinal gunshot wounds: Are "stable" patients really stable? World J Surg. 2002;26:1247-50.

9. Aggarwal R, Moorthy K, Darzi A. Laparoscopic skills training and assessment. Br J Surg. 2004;9I:I549-58. doi: IO.IOO2/bjs.48I6

Io. Wilson MR, Vine SJ, Bright E, Masters RS, Defriend D, Mcgrath JS. Gaze training enhances laparoscopic technical skill acquisition and multi-tasking performance: A randomized, controlled study. Surg Endosc. 20II;25:373I-9. doi: I0.1007/s00464-OII-I802-2

II. Danzer E, Dumon K, Kolb G, Pray L, Selvan B, Resnick AS, et al. What is the cost associated with the implementation and maintenance of an ACS/APDS-based surgical skills curriculum? J Surg Educ. 20II;68:519-25. doi: I0.IOI6/j.jsurg.20II.06.004

I2. Gershuni V, Woodhouse J, Brunt LM. Retention of suturing and knot-tying skills in senior medical students after proficiency-based training: Results of a prospective, randomized trial. Surgery. 20I3;I54:823-30. doi: IO.IOI6/j. surg.2013.07.016

I3. Xeroulis GJ, Park J, Moulton CA, Reznick RK, Leblanc V, Dubrowski A. Teaching suturing and knot-tying skills to medical students: A randomized controlled study comparing computer-based video instruction and (concurrent and summary) expert feedback. Surgery. 2007;I4I;442-9.

I4. Dimaggio PJ, Waer AL, Desmarais TJ, Sozanski J, Timmerman H, López JA, et al. The use of a lightly preserved cadaver and full thickness pig skin to teach technical skills on the surgery clerkship - a response to the economic pressures facing academic medicine today. Am J Surg. 20I0;200:I62-6. doi: Io.IoI6/j.amjsurg.2009.07.039

I5. Wongkietkachorn A, Rhunsiri P, Boonyawong P, Lawanprasert A, Tantiphlachiva K. Tutoring trainees to suture: An alternative method for learning how to suture and a way to compensate for a lack of suturing cases. J Surg Educ. 20I6;73:524-8. doi: Io.IoI6/j. jsurg.20I5.12.004

I6. Jowett N, Leblanc V, Xeroulis G, MacRae, H, Dubrowski, A. Surgical skill acquisition with self-directed practice using computer-based video training. Am J Surg. 20I7;193:237-42. doi: IO.IOI6/j.amjsurg.2006.II.003

I7. Fried GM, Feldman SL. Objective assessment of technical performance. World J Surg. 2008;156-6o.

I8. Scott DJ, Goova MT, Tesfay ST. A cost-effective proficiency-based knot-tying and suturing curriculum for residency programs. J Surg Res. 2007;I4I:7-I5.

\section{Apéndice 1 - Cuestionario}

\section{Estética}

I. Con respecto al color del tejido usado en la práctica, usted lo calificaría según una escala visual análoga de o a ı (siendo o muy desagradable y Io muy agradable), ¿en cuánto?

2. Con respecto a la consistencia del tejido usado en la práctica, usted lo calificaría según una escala visual análoga de o a Io (siendo o muy desagradable y io muy agradable), ¿en cuánto?

3. Con respecto a la textura del tejido usado en la práctica, usted lo calificaría según una escala visual análoga de o a Io (siendo o muy desagradable y Io muy agradable), ¿en cuánto?

\section{Sensación general}

I. Con respecto a la impresión general del tejido usado en la práctica, usted lo calificaría según una escala visual análoga de o a Io (siendo o muy desagradable y io muy agradable), ¿en cuánto?

\section{Utilidad}

I. Usted piensa que el tejido usado en esta práctica, ¿simula una sensación de tejido intestinal real?
a) Sí
b) No

2. ¿Permite el tejido usado, hacer una sutura simple con facilidad?
a) Mucho
b) Poco
c) Algo
d) Nada

3. ¿Permite el tejido usado, hacer una sutura continua con facilidad?
e) Mucho
f) Poco
g) Algo
h) Nada

4. ¿Permite el tejido usado, hacer el anudado intracorpóreo laparoscópico con facilidad?
i) Mucho
j) Poco
k) Algo
l) Nada 


\section{Actitud}

I. ¿Se encontraría a favor de tener más prácticas en este tipo de tejido?

- Muy a favor

- Algo a favor

- Poco a favor

- Nada a favor

2. De forma global, ¿cómo calificaría su experiencia del uso del tejido para la simulación de sutura y anudado laparoscópico? (Use un puntaje de o a Io, siendo o el puntaje más bajo y Io el puntaje más alto).

3. Del material usado, describa las características que más le agradaron y le permitieron hacer una mejor simulación de sutura y anudado laparoscópicos.

4. Del material usado, describa las características que limitaron o dificultaron el desarrollo de la práctica de simulación de sutura y anudado laparoscópicos. 